\title{
Management of chronic prostatitis/chronic pelvic pain syndrome
}

\author{
R. Christopher Doiron, MD; J. Curtis Nickel, MD, FRCSC
}

Department of Urology, Queen's University, Kingston, ON, Canada

Cite as: Can Urol Assoc J 2018;12(6Supp|3):S161-3. http://dx.doi.org/10.5489/cuaj.5325

\section{Introduction}

Prostatitis can refer to host of urologic diagnoses with a spectrum of etiologies and varying natural histories. Acute and chronic bacterial prostatitis have clear bacterial etiologies and are managed as infectious diseases. Chronic prostatitis/ chronic pelvic pain syndrome (CP/CPPS), characterized by chronic pelvic pain and voiding symptoms in the absence of a clear bacterial etiology, can be a more challenging entity to manage and will be the focus of this article. CP/CPPS is, after all, a common condition afflicting 2-6\% of men and is a common reason for referral to the community urologist. Furthermore, symptoms persisting for $>1$ year predicts for a dismal quality of life.

Several previously published guidelines describe the evaluation and management of CP/CPPS (see "Recommended reading"), however, recommendations based on level 3 and 4 evidence are pervasive, and the guidelines often fail to address the sometimes idiosyncratic approach required to manage this enigmatic condition. As such, physicians tend to manage patients based on a patchwork of what they learned during residency, experience, the last paper on the subject that they read, and perhaps a touch of evidence. We believe that successful management of CP/CPPS requires not only the best evidence, but also art, psychology, and black magic. The tips we provide, based on 25+ years of Prostatitis Research Clinic experience, should improve your approach for this enigmatic condition with less patient and provider frustration and significantly more hope for a better outcome.

\section{Practice tips}

Tip 1: First step in management is confirming diagnosis and clinical picture

A thorough and in-depth evaluation of patients being considered with a diagnosis of CP/CPPS is vital for success in their management. Given the heterogeneity of CP/CPPS patient presentations, a thorough of understanding of the

individual's clinical picture should be sought in order to be best equipped to succeed in developing an acceptable management plan. The approach we use in our clinic is outlined in a previous section of this supplement.

Tip 2: Develop individualized treatment plans that employ multimodal therapies

CP/CPPS patients are not a homogenous group of patients and this must be reflected in their management plans. Identifying individual phenotypes within the disease spectrum will help guide management, with various therapies directed at specific symptom complexes in a multimodal fashion (Fig. 1). Sequential monotherapy should be avoided, as CP/CPPS is too complex and this overly simplistic approach is destined for failure in too many patients.

\section{Tip 3: Use the "Five As" of CP/CPPS therapy}

\section{Avoidance}

Avoid dietary or physical activities (e.g., bike riding) that exacerbate symptoms.

\section{Antibiotics}

Some patients with CP/CPPS will respond to antibiotic therapy, particularly if they:

- Are naïve to antibiotics;

- Have a history of urinary tract infections (UTIs); or

- Have experienced a previous significant therapeutic response to antimicrobial therapy.

Once a four-week trial of antibiotic therapy fails, no further antibiotics should be prescribed unless a true UTI is documented.

\section{Alpha-blockers}

Consider as part of a multimodal treatment regime, particularly in those with (obstructive) voiding symptoms. 


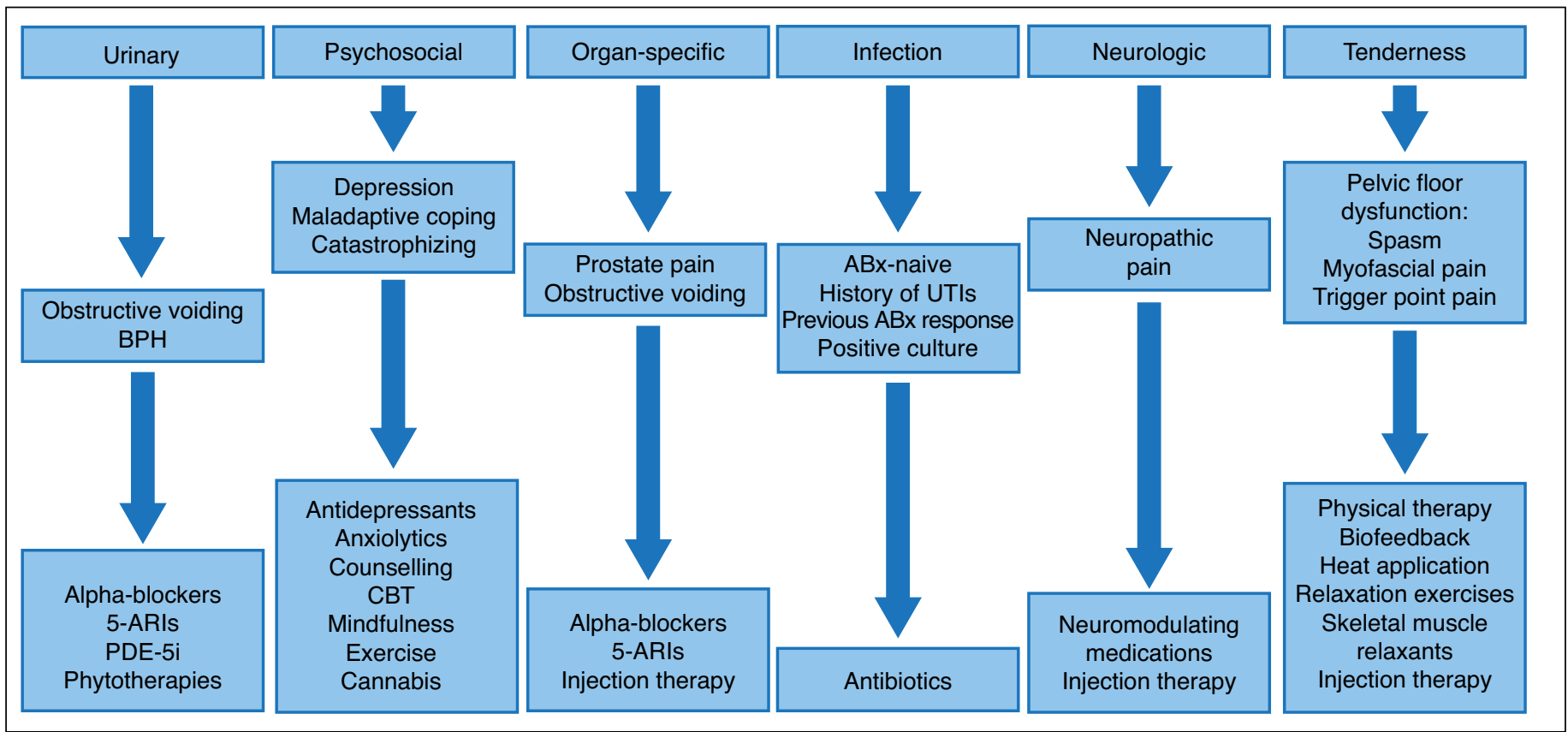

Fig. 1. A multimodal approach to treatment, directed at specific symptom patterns, organized here using the UPOINT phenotype approach. ABx: antibiotic; $B P H$ : benign prostatic hyperplasia; CBT: cognitive behavioural therapy; PDE-5i: phosphodiesterase type 5 inhibitor; UTI: urinary tract infection; 5 -ARI: $5 \alpha$-reductase inhibitors.

\section{Anti-inflammatories}

Should also be considered as part of a multimodal treatment plan, as some patients will experience benefit.

\section{5-alpha reductase inhibitors}

These medications should only be considered in men $>50$ years old with CP/CPPS and enlarged prostates (12-16\% of men with CP/CPPS also have benign prostatic hyperplasia $[\mathrm{BPH}])$.

\section{Tip 4: Consider phytotherapies}

There is little downside to considering phytotherapy (particularly quercetin and cernilton) as part of one's multimodal treatment plan. There are few significant side effects (apart from cost) to consider and they have been shown to be more efficacious than placebo in randomized controlled studies.

\section{Tip 5: Physical therapy can be helpful}

For patients who exhibit pelvic floor dysfunctional pain spasm, myofascial pain, trigger point pain - physical therapy regimens have been shown to very effective. Treatment regimens may include specific pelvic floor physiotherapy, biofeedback, local heat application, and the teaching of relaxation exercises. We encourage urologists to seek out collaboration with their local physiotherapist with an interest in treating the pelvic floor. Skeletal muscle relaxants, e.g., diazepam (oral or suppositories), baclofen, or cyclobenzaprine may be helpful in this phenotype.

\section{Tip 6: Injection therapy works for selected patients}

Injection of local anesthetic for trigger point pain or localized myofascial pain is an important consideration for selected patients. Repeat ilioinguinal injections can downregulate or desensitize neuropathic pain seen in orchodynia. Periprostatic blocks (similar to that used for transrectal ultrasound-guided prostate biopsy) can be helpful in patients with real prostate pain (organ-specific, usually described like sitting on a "golf ball"). If pudendal neuropathy has been identified - unilateral pain localized to the perineum that is characteristically relieved with sitting on toilet seat or ring pillow - a pudendal nerve block may be particularly helpful. Pelvic floor, periprostatic, and pudendal nerve injection therapy can be offered by the urologist in the office using a transrectal or transperineal approach following appropriate training in this methodology.

\section{Tip 7: Recognize maladaptive psychological coping behaviours}

Depression is a frequent comorbid condition in those with $\mathrm{CP} /$ CPPS and should be addressed with assistance from the family doctor or psychiatrist. Catastrophizing - rumination, magnification, and helplessness stages - will impede progress in CP/ CPPS patients and requires treatment. Cognitive behavioural 
therapy or mindfulness training are options that may help these patients. Furthermore, resting as a coping mechanism is important to avoid. Patients need to be encouraged to avoid rest as treatment and instead use exercise as part of their treatment plan. Low-impact exercises - walking, swimming, elliptical, yoga - can be particularly helpful in these patients.

\section{Tip 8: Neuropathic pain may require neuromodulating medications}

There are various options for the urologist to treat neuropathic pain, including amitriptyline, gabapentinoids (or even the combination), and acupuncture. These interventions may be helpful as part of a multimodal approach in those with resistant symptoms.

\section{Tip 9: Sexual symptoms should be addressed}

CP/CPPS may manifest in sexual dysfunction, either associated with ejaculatory pain or in association with psychological sexual dysfunction. Standard erectile dysfunction strategies should be employed, including use of phosphodiesterase type 5 (PDE-5) inhibitors (daily tadalafil), which have been shown to be helpful in alleviating urinary symptoms and pain in CP/CPPS patients, as well as addressing their erectile dysfunction. Sexual counselling may also be helpful in select patients.

\section{Tip 10: Avoid opioids}

Opioids are generally not effective as a long-term pain management strategy for patients with CP/CPPS and should be considered as short-term flare therapy in very carefully selected patients or a treatment of last resort when all else fails. Once opioid therapy is initiated, poor long-term outcomes can be expected.

\section{Tip 11: Cannabis may improve quality of life}

Marijuana does not have a big impact on pain, but does seem to help coping and may improve general quality of life and promote increased physical activity in some men with CP/CPPS.

\section{Tip 12: Work with your chronic pain clinic}

One may consider collaboration with the local pain clinic. Urologists should be aware of practice patterns at their local centre and the preponderance for pain clinics to treat with non-pelvic injection therapy and opioids in their management plans. However, they may be extremely helpful in coordinating referral to psychology, social work, and physiotherapy.

\section{Final tip}

The final and perhaps the most important lesson or tip we have learned in all our years of evaluating and treating thousands of CP/CPPS patients is that both the urologist and the patient must have realistic expectations. This understanding is an important cornerstone of managing CP/CPPS patients. The urologist cannot expect to cure this chronic disease and patients must understand that we do not offer cure (although Mother Nature can and does provide cure for some of our patients). Understanding, empathy, and a patient-centred plan to improve symptoms, increase physical activities, and improve quality of life must be the focus of our management strategy.

Competing interests: Dr. Nickel has been a consultant for Astellas, Auxillium, Eli Lilly, Farr Labs, Ferring, GSK, Pfizer, Redleaf Pharma, Taris Biomedical, Tribute, and Trillium Therapeutics; a lecturer for Astellas and Eli Lilly; and has participated in clinical trials supported by Eli Lilly, GSK, J\&J, Pfizer, and Taris Biomedical. Dr. Doiron reports no competing personal or financial conflicts related to this work.

This paper has been peer reviewed.

\section{Recommended reading}

1. Nickel JC. Prostatitis. Can Urol Assoc J 2011;5:306-15. https://doi.org/10.5489/cuaj.686

2. Engeler D, Baranowski AP, Borovicka J. et al. EAU guidelines on chronic pelvic pain. Pocket Guidelines, 2017 Edition, European Association of Urology, 2017: Pg 352-374.

3. Nickel IC, Shoskes DA, Wagenlehner FME. Management of chronic prostatitis/chronic pelvic pain syndrome (CP/CPPS): The studies, the evidence, and the impact. World J Urol 2013;31:747-53. https://doi. org/10.1007/s00345-013-1062-y

Correspondence: Dr. R. Christopher Doiron, Department of Urology, Queen's University, Kingston, ON, Canada; chris.doiron@queensu.ca 